\title{
Plastinación y Descripción Anatómica de Hígado, Bazo, Estó- mago y Riñones del Delfín Nariz de Botella (Tursiops truncatus)
}

\author{
Plastination and Anatomy Description of Organs of the Bottlenose Dolphin (Tursiops truncatus)
}

Gabriela Sánchez Fabila*; María Dolores Contreras Villanueva* \& Roberto Moreno Colín*

SÁNCHEZ, F. G. \& CONTRERAS, V. M. D. \& MORENO, C. R Plastinación y descripción anatómica de hígado, bazo, estómago y riñones del delfín nariz de botella (Tursiops truncatus). Int. J. Morphol., 34(2):644-652, 2016.

RESUMEN: Se plastinaron y describieron cuatro diferentes órganos internos de delfín nariz de botella (Tursiops truncatus) obteniendo modelos didácticos, perdurables gracias a la técnica de plastinación realizada en ellos, haciéndolos inodoros, no tóxicos y manipulables que permiten el estudio de los órganos internos como son el estómago, riñones e hígado de T. truncatus, contribuyendo a los trabajos de anatomía no patológica en delfines que son de importancia ecológica, turística y económica para nuestro país. Las descripciones anatómicas permiten avanzar en el conocimiento sobre los órganos internos, y si bien es cierto que se ha realizado un esfuerzo por estudiarlos existen pocos trabajos anatómicos realizados en ellos. Siendo este artículo una contribución al estudio de estos cetáceos.

PALABRAS CLAVE: Plastinación; Delphinidae; Nariz de botella; Órganos; Descripción.

\section{INTRODUCCIÓN}

La técnica de plastinación es una técnica innovadora que permite preservar órganos y organismos de distintas especies para su posterior estudio, su proceso consiste en el remplazo de los fluidos remanentes en un cuerpo por medio de un polímero. Esta técnica fue ideada en 1977 por el Dr. Gunther von Hagens, y siendo modificada por biólogos de la FES Iztacala UNAM, teniendo como resultado el modelo final obtenido con esta técnica conserva flexibilidad y un color realista sin olor ni toxicidad, otras de las ventajas que presenta el preservar órganos con esta técnica es el disponer de los modelos para poder impartir una clase, donde los materiales se podrán no solo observar sino también manipular con la confianza de ser durables y limpios sin riesgo para el maestro o los estudiantes a diferencia de preparaciones convencionales que al poco tiempo se tienen que desechar y sustituir (Bravo, 2006).

Esta técnica nos ayuda a comprender y describir mejor la anatomía interna de los organismos ya que su preservación nos permite estudiar las complejidades de algunos órganos y su funcionamiento pues ahora lo analizamos de manera tridimensional observando sus dimensiones, coloraciones y sus planos morfofuncionales, en sus respec- tivas posiciones e interrelaciones, actividad que se realizaba arduamente con el apoyo de bibliografía, imágenes fotográficas, que al ser planas nos limitaban nuestro estudio aun siendo de buena calidad (Reyes Aguilar, 2007); mientras que dichas muestras plastinadas permiten el arreglo de todos los componentes del tejido para ser estudiados en un contexto inalterado (Von Hagens et al., 1987).

Para el presente estudio se trabajó con mamíferos debido a muchas de sus características adaptativas (CastellóOrvay, 1976). Especialmente el orden de los Cetáceos qué está formado por un conjunto de mamíferos adaptados a la vida marina, gracias a la estructura y función de su cuerpo adquiridas a lo largo del camino evolutivo (Castelló-Orvay).

Todos son por completo acuáticos; la caja torácica alberga y protege a los órganos internos como el aparato digestivo que también presenta adaptaciones a la vida en el mar (Vaughan, 1988).

El aparato circulatorio de los cetáceos es el típico de los mamíferos con el añadido de una rete mirabilis intercalada en el trayecto de las arterias (Castelló-Orvay). Se ob- 
serva un estómago anterior, un estómago medio y finalmente un estómago pilórico (Vaughan).

Con respecto al aparato excretor se sabe que los cetáceos presentan riñones de gran tamaño y aspecto granuloso; cada uno de estos gránulos es una pequeña esfera con funcionamiento semejante al de un riñón individual (Vaughan).

Los Cetáceos en términos de abundancia, diversidad de especies y distribución son de los grupos más importantes de mamíferos acuáticos (Vaughan), siendo la familia Delphinidae el grupo más abundante y diverso. En nuestro país contamos con Tursiops truncatus es una especie cosmopolita distribuida en todos los océanos templados y tropicales del mundo siendo la especie de mamífero marino costal más común en el Golfo de México (Blaylock et al., 1995).

\section{MATERIAL Y MÉTODO}

Se trabajó con 6 órganos internos del delfín $T$. truncatus (un hígado, tres riñones, un bazo y un estómago) colectados y etiquetados por el Dr. Alberto Delgado Estrella y el Biol. Arturo Romero Tenorio gracias a un convenio de colaboración entre la Facultad de Estudios Superiores Iztacala U.N.A.M y el Instituto Vía Delphi para la Investigación de los Mamíferos Acuáticos A.C. (del parque ecológico Xcaret).

Dichas muestras se recibieron directamente en el Laboratorio de Anatomía Animal Comparada (FES-I) por medio de paquetería en contenedores de plástico envueltos e impregnados con formol. Los órganos se lavaron en agua corriente dentro de cubetas para en seguida tomar las medidas morfométricas de cada uno; posteriormente fueron sumergidos en formaldehído al $4 \%$ durante 7 días en recipientes de plástico a temperatura ambiente con el fin de fijarlos y evitar su descomposición.

Deshidratación. Pasado este periodo se sacaron del formol y se sumergieron en agua corriente durante dos días con la finalidad de comenzar el proceso de deshidratación, en el cual se sacaron del agua y se sumergieron en acetona al 100 $\%$ durante un mes a una temperatura de $-30^{\circ} \mathrm{C}$; durante este tiempo se realizaron varios cambios de acetona con el fin de eliminar residuos de grasa y agua del órgano, siendo remplazada nuevamente por acetona pura.

Impregnación. Una vez pasado el mes se inició el proceso impregnación pasiva, realizada gradualmente con los órganos saturados en acetona llevándolos a resina poliéster mc-
40 en dos etapas que duraron de uno a tres meses cada una a temperatura ambiente:

1a) Inmersión en una solución 1:1 de resina y acetona (en cubetas de plástico con tapa).

2a) Impregnación en resina $100 \%$ (en frascos de vidrio).

Secado. Cumplido este lapso se removió el exceso de resina lavando los órganos en acetona pura, una vez secos se rociaron con esmalte acrílico de secado rápido dándoles de 2 a 3 aplicaciones con un periodo de secado de 3 a 15 días. Finalmente plastinados los distintos órganos se tomaron nuevamente sus medidas para iniciar las descripciones anatómicas.

\section{RESULTADOS}

Fueron obtenidas las medidas morfométricas de los órganos internos del delfín nariz de botella (T. truncatus) antes y después del proceso.

Con las medidas morfométricas se calculó el porcentaje de deformación de cada órgano (Tabla I) por medio de la siguiente fórmula:

$$
\% \text { de formación }=\frac{y_{0}-y_{f}}{y_{0}} \times 100
$$

En donde:

$\mathrm{y} 0=$ medida inicial

$\mathrm{yf}=$ medida final

Se observó la retención de varias características posteriores al proceso en el hígado, riñones y bazo, como: color negruzco, con rigidez, estructura anatómica normal, tamaño, flexibilidad y detalle de superficie normal, solo en el estómago el color fue seminegruzco.

El detalle de superficie se refiere a la calidad del aspecto externo del órgano una vez plastinado mientras que la estructura anatómica se relaciona con la conservación de la morfología de los especímenes después de la plastinación (Von Hagens et al.).

En todos los casos se observó un cambio de tonalidad de rosado/rojizo a café obscuro que ocurrió durante el proceso de impregnación pasiva (Figs. 1-3); así mismo, se advierte la reducción del tamaño original de los órganos de $4 \%$ hasta $11 \%$ de largo y de $6 \%$ hasta $16 \%$ de ancho 
Tabla I. Cálculo del porcentaje de deformación de los órganos.

\begin{tabular}{lcc}
\hline Órgano & Largo & Ancho \\
\hline Hígado & $9 \%$ & $9 \%$ \\
Riñón 1 & $9 \%$ & $12 \%$ \\
Riñón 2 & $7 \%$ & $16 \%$ \\
Riñón 3 & $8 \%$ & $8 \%$ \\
Bazo & $8 \%$ & $11 \%$ \\
Estómago & $10 \%$ & $9 \%$ \\
\hline
\end{tabular}

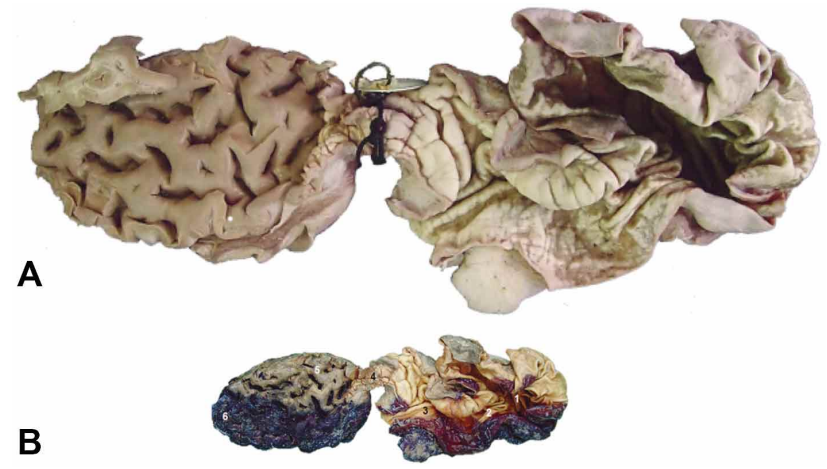

Fig. 1. Estómago de T. truncatus fijado en formol $4 \%$ (A) y plastinado (B).

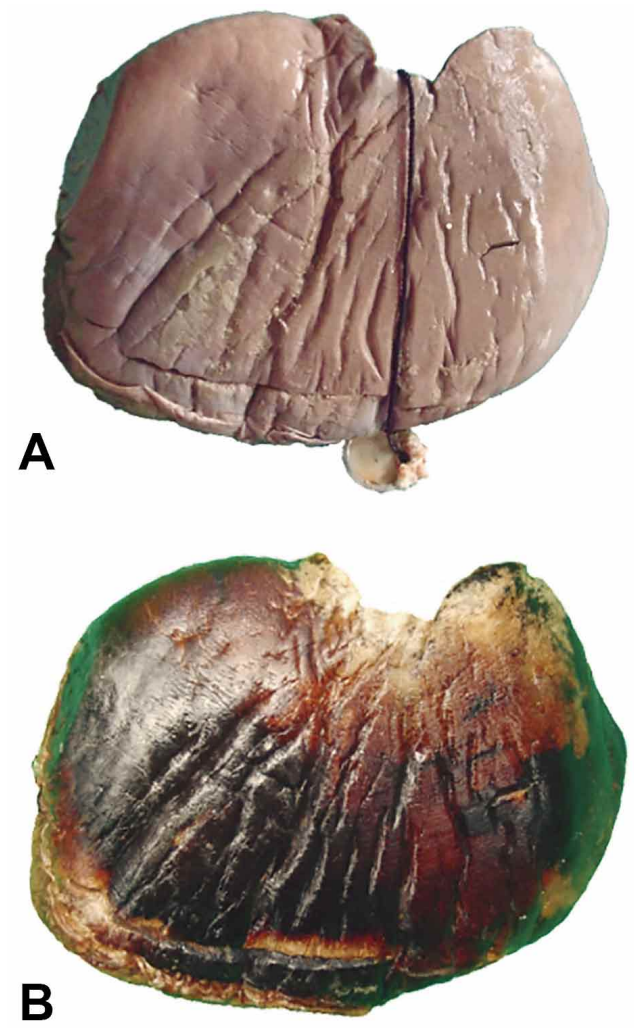

Fig. 2. Hígado de T. truncatus fijado en formol 4 $\%$ (A) y plastinado (B).
(Tabla I). En cuanto a la forma cabe mencionar que todos los órganos conservaron su aspecto original (Figs. 1-3), y pese a que adquirieron notable rigidez mantuvieron una textura cercana a la del órgano en fresco.

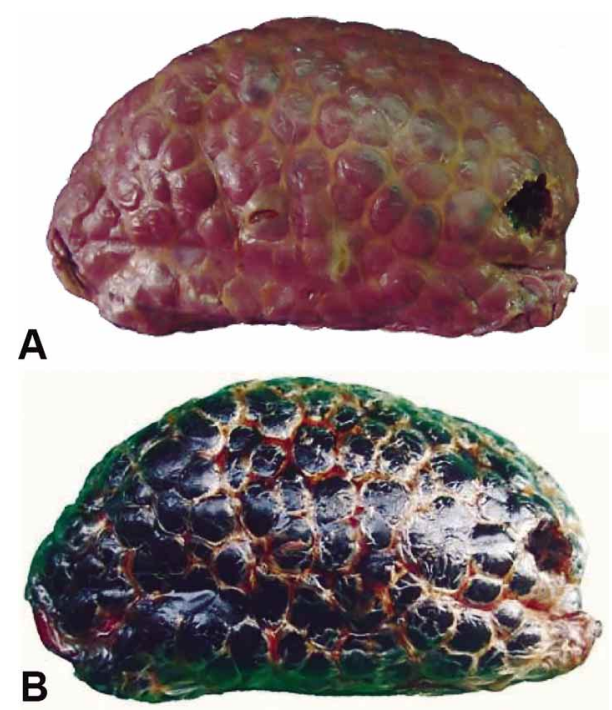

Fig. 3. Riñón \#1 de T. truncatus fijado en formol $4 \%$ (A) y plastinado (B).

Descripciones anatómicas de cada órgano de región cefálica a caudal:

Hígado: Presenta forma semiovoidea, de $26,5 \mathrm{~cm}$ de longitud transversal, $13,8 \mathrm{~cm}$ en sentido dorso ventral y un peso de $945 \mathrm{~g}$.

Su cara diafragmática es convexa en toda su extensión (Fig. 4A); sin embargo, presenta una depresión en su parte superior llamada impresión cardiaca, ya que es causada por la presencia de corazón y pericardio, con los que se relaciona por intermedio del diafragma. En la parte derecha, la cara diafragmática es fuertemente convexa, se relaciona con el diafragma, al adaptarse a la columna vertebral, la parte posterior describe una amplia concavidad transversal.

La cara visceral corresponde a la superficie del corte del semiovoide, es más o menos plana y se orienta en sentido dorsocaudal y un poco a la izquierda. Hacia la unión de sus dos tercios izquierdos presenta la fisura del ligamento redondo un surco dorsoventral, que aloja al ligamento redondo (Fig. 4B) y termina adelante en la fisura del ligamento redondo que interrumpe al margen inferior. Este surco corresponde a la inserción del ligamento falciforme (Fig. 4B) en la cara diafragmática; entre ambos dividen al hígado en lóbulos derecho e izquierdo. A la derecha de este surco, 
casi en el centro, la cara visceral presenta otro surco más ancho, formado por dos segmentos: uno ventral, iniciado en el margen inferior, que se llama fosa de la vesícula biliar (fosa cística); y otro dorsal, que es una prolongación del surco de la vena cava.

El lóbulo izquierdo presenta en toda su superficie una ligera excavación, recibe el nombre de impresión gástrica (Fig. 4B) debido a que en su mayor parte se relaciona con la cara anterior y fondo del estómago.

El lóbulo derecho está ocupado por tres depresiones amplias y poco profundas causadas por las vísceras vecinas. La ventral es la impresión cólica, la media es la impresión renal y la posterior es la impresión suprarrenal, que se continúa dorsalmente con el área desnuda (Fig. 4B).

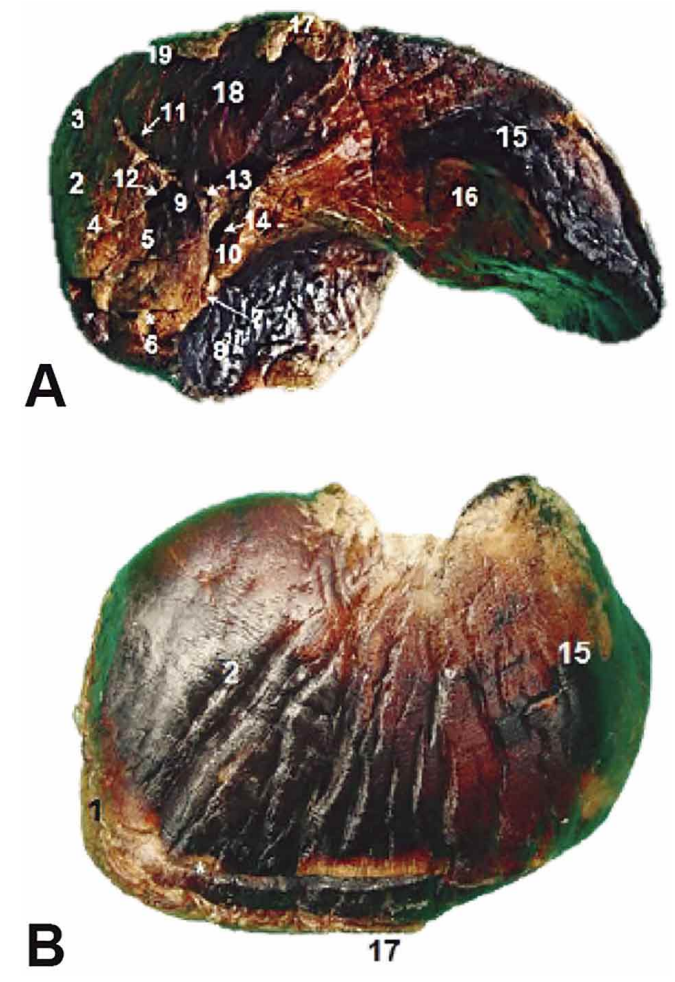

Fig. 4. (A) Cara anterior y (B) vista posteroinferior del hígado, en donde: (1) ligamento falciforme; (2) lóbulo derecho; (3) impresión cólica; (4) impresión renal; (5) impresión duodenal; (6) impresión suprarrenal; (7) hoja inferior del ligamento coronario; (8) área desnuda; (9) proceso caudado; (10) proceso papilar; (11) conducto hepático común; (12) vena porta; (13) arteria hepática; (14) cava inferior; (15) lóbulo izquierdo; (16) impresión gástrica; (17) ligamento redondo; (18) lóbulo cuadrado y (19) margen inferior.
Por su parte el lóbulo cuadrado (Fig. 4B) presenta una superficie ligeramente convexa, su margen posterior forma el labio ventral de la puerta hepática y a menudo se prolonga, por medio de un puente de tejido hepático que lo une al lóbulo izquierdo, cubriendo al ligamento redondo.

El margen inferior (Fig. 4B), más o menos agudo, se dirige hacia abajo y a la derecha (Fuentes Santoyo, 2006).

Bazo. Aplanado y oblongo, con 8,0 cm de largo por 5,6 $\mathrm{cm}$ de ancho y un peso de 26,3 g. Posee tres depresiones y dos márgenes (el superior un poco plegado hacia la cara posterior). Se le describen dos caras. En la cara anterior (diafragmática por tener relación con tal músculo), lisa y convexa, se pude apreciar la muesca o mella de $0,97 \mathrm{~cm}$ (Fig. 5A), mientras que en la cara posterior (visceral), cóncava y lisa, se localizan las tres depresiones: impresión gástrica, renal y cólica, así como restos de la cola del páncreas y de los ligamentos gastroesplénico y frenoesplénico (Fig. 5B.).

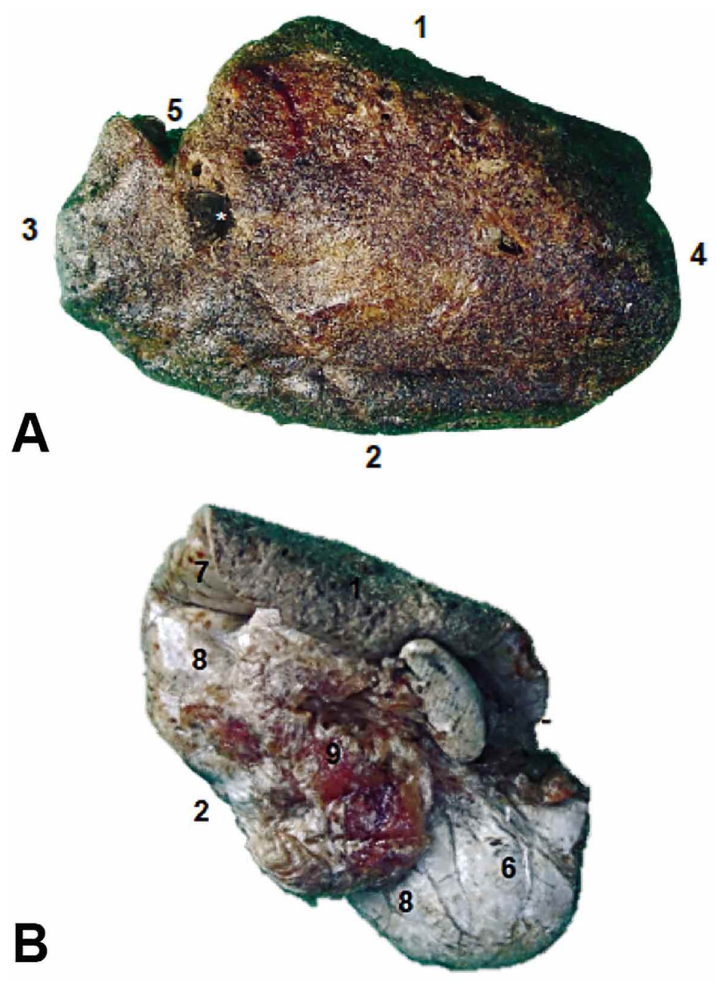

Fig. 5. (A) Caras anterior y (B) posterior del bazo, en donde: (1) margen superior; (2) margen inferior; (3) miembro anterior; (4) miembro posterior; (5) muesca; (6) impresión cólica; (7) impresión gástrica; (8) impresión renal y (9) restos de la cola del páncreas y ligamentos gastroesplénico y frenoesplénico. 
El bazo es el órgano linfoide más grande, y principalmente está constituido por las células responsables de la respuesta inmunitaria: los linfocitos y las células derivadas de ellos, como son las células plasmáticas. Está situado en la circulación sanguínea, por lo que no tiene vasos linfáticos aferentes. Realiza las funciones de producción de linfocitos y anticuerpos, filtración de la sangre, y destrucción de células sanguíneas, principalmente hematíes.

Dos partes se distinguen en el bazo: la pulpa roja formada principalmente por el componente sanguíneo (senos venosos y cordones de Billroth) y la pulpa blanca formada por el componente linfoide (Boya, 2004).

La función del bazo en Cetáceos sigue siendo controvertida. De hecho, el pequeño tamaño de este órgano, junto con su típica forma globular y superficie lisa, pueden argumentan en contra de su función de almacenamiento de sangre. Además, la presencia de megacariocitos sugiere un papel en la hematopoyesis extramedular (Colangelo et al., 2006).

Estómago. Pesó 432 g con una longitud total de $34,2 \mathrm{~cm}$. Se divide en las siguientes cámaras:

1. Ante-estómago (Fig. 6A): también llamado estómago esofágico, con 10,6 cm de largo por 15,3 cm ancho. Cámara ubicada entre el esófago y el estómago principal, su capa muscular es distensible, prominente y gruesa, funciona como un compartimento de soporte. Alguna actividad digestiva se lleva a cabo aquí debido al reflujo de jugos gástricos del estómago principal. Es homóloga al ante-estómago de ungulados (Mead, 1993, 2007).

2. Estómago principal (Fig. 6A): también llamado estómago cardiaco o estómago secundario; cámara digestiva activa; con una longitud de $7,7 \mathrm{~cm}$ por $12,1 \mathrm{~cm}$ de ancho. Su capa muscular es relativamente delgada. Es la porción secretora activa del complejo estomacal, produciendo moco, enzimas digestivas y ácido hidroclorhídrico. Se comunica con los canales de conexión por una pequeña apertura que puede ser cerrada por acción muscular (Mead, 1993, 2007).

3. Cámaras de conexión (Fig. 6B): también llamadas canales de conexión, mide 4,3 cm de largo y 2,6 cm de ancho; conoci- do también como estómago intermedio o estómago terciario. Se trata de un paso estrecho y tortuoso, provisto de una válvula y esfínter en ambos extremos y usualmente un tercero en el medio. Se comunican con el compartimento pilórico por un estrecho esfínter ubicado en la pared distal de la última cámara de conexión; y a menudo es ligeramente evertido dentro del compartimento pilórico. Su función en la mayoría de los cetáceos parece ser valvular (Mead, 1993, 2007).

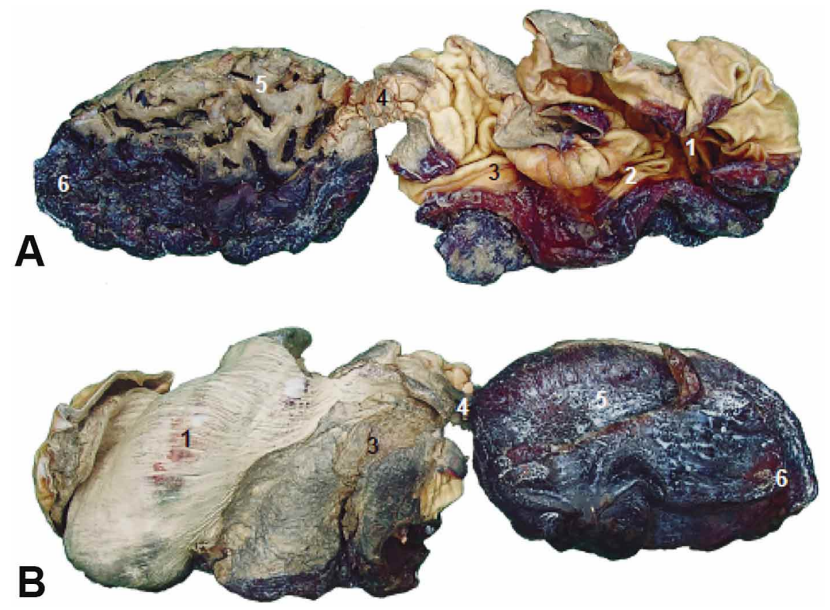

Fig. 6. Vista de la apariencia interna del estómago, en donde: (1) Ante-estómago; (2) apertura entre el ante-estómago y el estómago principal; (3) estómago principal; (4) cámaras de conexión; (5) compartimentos pilóricos y (6) dilatación proximal del duodeno.

4. Compartimentos pilóricos (Fig. 6 A and B): también llamados tercero/cuarto estómago, estómago distal y estómago pilórico, mide $11,6 \mathrm{~cm}$ de largo por 10,2 cm de ancho. $\mathrm{Su}$ pared muscular es más delgada que cualquiera de los otros compartimentos. Se comunica con la ámpula duodenal por un esfínter pilórico. En la mayoría de los cetáceos parece ser una cámara de sostén y neutralización para la comida parcialmente digerida (Mead, 1993, 2007).

Riñones. Se describen 3 riñones, difiriendo cada cual en cuanto a forma y medidas (Tabla II). Presentan dos caras, anterior y posterior, un margen lateral convexo y un margen medial cóncavo en su centro, el cual alberga una muesca llamada hilio renal, así como dos polos redondeados, superior e inferior (Fig. 7A).

Tabla II. Datos obtenidos de 3 riñones de T. truncatus.

\begin{tabular}{|c|c|c|c|c|c|c|c|c|c|c|}
\hline \multirow{2}{*}{ Riñón } & \multirow{2}{*}{ Figura } & \multirow{2}{*}{ Posición } & \multirow{2}{*}{$\begin{array}{l}\text { Largo } \\
\text { (cm) }\end{array}$} & \multirow{2}{*}{$\begin{array}{l}\text { Ancho } \\
\text { (cm) }\end{array}$} & \multirow{2}{*}{$\begin{array}{l}\text { Peso } \\
\text { (g) }\end{array}$} & \multirow{2}{*}{$\begin{array}{l}\text { Número de } \\
\text { Renículos }\end{array}$} & \multicolumn{2}{|c|}{$\begin{array}{c}\text { Renículo más } \\
\text { pequeño }\end{array}$} & \multicolumn{2}{|c|}{$\begin{array}{l}\text { Renículo más } \\
\text { grande }\end{array}$} \\
\hline & & & & & & & $\begin{array}{c}\text { Largo } \\
\text { (cm) }\end{array}$ & $\begin{array}{c}\text { Ancho } \\
\text { (cm) }\end{array}$ & $\begin{array}{c}\text { Largo } \\
\text { (cm) }\end{array}$ & $\begin{array}{c}\text { Ancho } \\
\text { (cm) }\end{array}$ \\
\hline 1 & 55,56 & Izquier da & 12,5 & 9,0 & 88,9 & 220 & 0,50 & 0,45 & 1,15 & 0,33 \\
\hline 2 & 57 & Derecha & 12,6 & 8,2 & 111,5 & 284 & 0,10 & 0,07 & 1,07 & 0,27 \\
\hline 3 & 58 & Derecha & 23,0 & 12,6 & 361,3 & 300 & 0,50 & 0,37 & 1,50 & 1,14 \\
\hline
\end{tabular}


Rodeados por la cápsula adiposa renal y la fascia renal, se observan en cantidad desigual (Tabla II), una serie de renículos empaquetados firmemente (Fig. 7B), que colectivamente forman cada riñón.

En el riñón \#2 (Fig. 8) se observa al uréter, el cual se aleja del hilio renal.
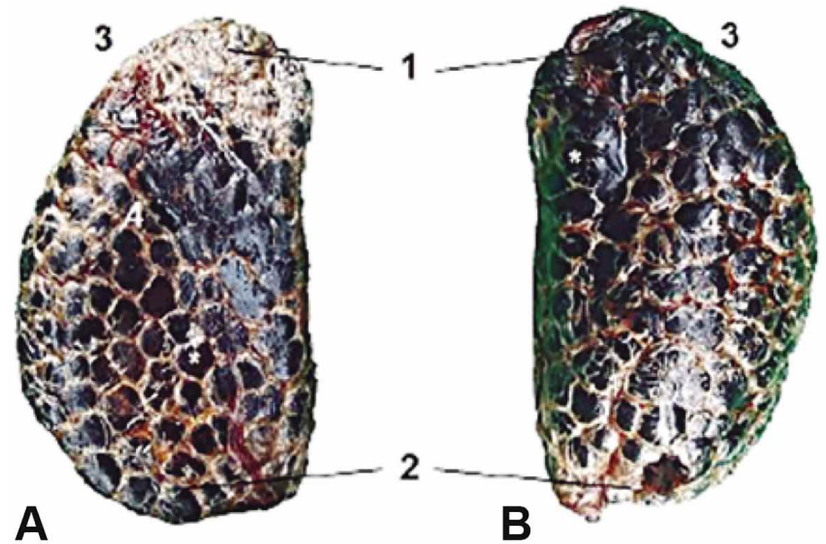

Fig. 7. Riñón \#1: (A) vistas posterior y (B) anterior, en donde: (1) polo superior; (2) polo inferior; (3) margen medial y (4) margen lateral.
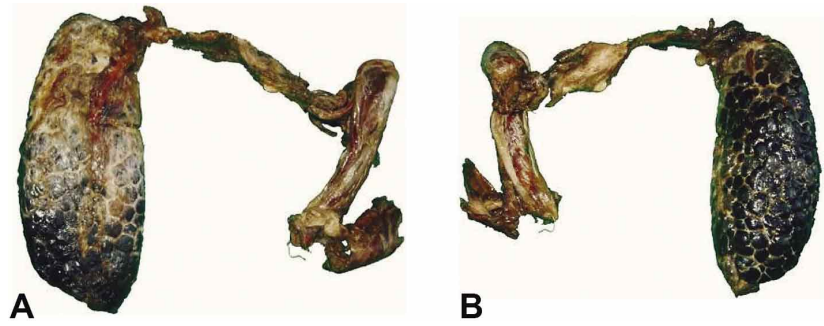

Fig. 8. Riñón \#2: (A) vista posterior; (B) vista anterior.

\section{DISCUSIÓN}

Se aplicó la técnica de plastinación modificada en algunos órganos internos de delfín nariz de botella utilizando resina poliéster mc-40 y como anotaba Von Hagens et al. los especímenes resultantes con este tipo de resinas son opacos y firmes pero no irrompibles, técnica adecuada para elaborar piezas de demostración como las del presente estudio.

Las piezas biológicas obtenidas ofrecen las ventajas de ser duraderas, almacenables y manipulables en seco sin alguna medida preventiva, con forma y volumen natural, textura aproximada a la real, inodoros y no desprenden vapores tóxicos. Dichos especímenes no precisan de tratamientos especiales para su mantenimiento y se pueden mantener limpios por métodos tradicionales.
Los órganos se describieron destacando su estructura específica, permitiendo el estudio de la función anatómica y textura que suelen perderse con la técnica de preservación típica en formol. Pese a que los órganos plastinados mantuvieron sus formas y estructuras en la posición anatómica esperada, las desventajas recaen en que sus dimensiones se redujeron de manera poco significativa, perdieron flexibilidad y color original del mismo modo que se reporta en los trabajos de Silva Pérez (2007) y Ali \& Al-Thnaian (2007).

Así mismo, los órganos plastinados probablemente perdieron su color original durante el proceso debido a que durante la acción del polímero y la acetona, los pigmentos de los cromatóforos fueron expuestos y por consiguiente, diluidos como señalara Silva Pérez. Del mismo modo al explicar este ennegrecimiento deben ser consideradas tanto la oxidación de la hemoglobina como la posibilidad de que sea resultado del índice de refracción de la resina que Von Hagens \& Whalley (2009) destacaron acerca de sus primeros ensayos sobre plastinación. Lo anterior tomando también en cuenta que la fijación es un paso fundamental que no se llevó a cabo de primera instancia en el LAAC, debido a que los órganos fueron enviados envueltos en gasas impregnadas en formol; y ya Miklosová \& Miklos (2004) mencionan que los órganos que no alcanzan a impregnarse en su totalidad tienden a encogerse adquiriendo un color obscuro. Todo esto ligado a la omisión del proceso de curado (Peroxido de metil etil cetona y acetona), durante el cual la emulsificación atenúa el color del espécimen.

La forma de los órganos se mantuvo ya que los especímenes no mostraron diferencias perceptibles en la calidad del detalle de superficie, aunque se produjo una reducción dimensional acaso atribuible al efecto de fijación y deshidratación y quizá a un rápido permeado del espécimen en un solo baño de resina/acetona antes de entrar a resina pura.

La pérdida de flexibilidad de los tejidos se imputa al uso de resina poliéster mc-40 en lugar de alguno de los silicones BIODUR $^{\circledR}$ típicamente utilizados para la técnica estándar de plastinación, esto debido a que como mencionan Daviau et al. (1997) es necesario contar con un permiso para realizar la técnica con los materiales originalmente dispuestos para este fin. Ya Von Hagens et al. señalaron que los especímenes plastinados con copolímeros epoxi-silicona resultan lo suficientemente rígidos como para ser pulidos pero no son irrompibles; aunque plastinar con este tipo de resinas ofrece la ventaja práctica de poder llevar a cabo la impregnación forzada a temperatura ambiente. Todo esto considerando del mismo modo lo anotado por Ripani et al. (1994) acerca de que el tiempo de deshidratación debe ser el menor posible, para prevenir la fragilidad y pérdida de flexibilidad de los especímenes. 
Las estructuras de cada órgano se mantuvieron tal como en los esquemas consultados para su descripción, no obstante, al no haber obtenido los órganos del delfín dentro del LAAC, hay estructuras que no se presentan en la descripción. La única dificultad durante la descripción anatómica inherente al proceso de plastinación se debió al cambio de coloración del órgano que en ocasiones impedía distinguir claramente las estructuras. Otro obstáculo que se presentó fue que algunos órganos tenían ciertas estructuras plegadas incluso antes de comenzar con la técnica (y por tanto ajeno al proceso), ocultando así otras secciones del órgano.

Se presentaron algunos incidentes durante el plastinado de los corazones y el estómago, siendo que la resina se polimerizó antes del término del proceso. Lo anterior sucedió estando los órganos en solución resina/acetona (1:1) y en resina pura; y esto pudiera explicarse tomando en cuenta que la impregnación a temperatura ambiente limita el tiempo de vida de la resina, debido a que la viscosidad del polímero se incrementa después de algunas semanas; y por otra parte, se reduce el tiempo de impregnación como apuntan Ripani et al. Este problema pudiera resolverse con la sugerencia de Kularbkaewi et al. (1996) mediante la mezcla del polímero con acetona antes de rehusarlo en un nuevo baño de especímenes.

Debido a estos percances los órganos se retiraron de las soluciones en las que se encontraban y se llevaron a un baño de acetona (4 días en el caso de los corazones y 6 en el del estómago), durante el cual se fueron eliminando manualmente los trozos de resina sobrante, cuando fue necesario esto se hizo con ayuda de una fresadora (Dremel) para el corazón \#2, así como lijas bastardas y aguja de disección en el estómago, hasta limpiar casi en su totalidad los órganos antes mencionados.

Como mencionan Donaldson (1977) y Sokolov \& Volkova (1973) en relación con lo referente a la lengua de $T$. truncatus, una de sus características más notables es la ausencia de papilas filiformes, fungiformes, foliadas o circunvaladas presentes en la mayoría de los mamíferos, por lo que podría ser que el gusto sea un sentido quimiorreceptor en Odontocetos. De la misma forma que este autor describe, en el presente trabajo también se hallaron surcos terminales que anteriormente no se le describían a T. truncatus. El mismo autor ya señala que en estudios anteriores se advierte la existencia de algunos quimiorreceptores que pudieran funcionar como receptores gustativos, siendo la raíz de la lengua el lugar más probable para encontrarlos.

Ahora bien, la presencia de remanentes de papilas marginales sugiere lo apuntado por Kastelein \& Dubbeldam (1990) en lo referente a que dichas papilas tienen la función de impe- dir la filtración de leche hacia la cavidad oral durante el periodo de lactancia así como para evitar que el agua expulsada al ingerir el alimento vuelva a ingresar a la cavidad oral cuando el delfín comienza a alimentarse de peces. Dichas papilas reducen su tamaño con el paso del tiempo, por lo cual no se observan tan claramente en el espécimen plastinado. Otros mecanismos para limitar el paso del agua hacia el interior pueden ser encontrados como anota Donalson, en la acción conjunta de los músculos estilogloso, hiogloso y longitudinal inferior que parecen permitirle a la lengua ayudar a sellar la orofarínge; así como en la observación de que los delfines pueden expulsar enérgicamente chorros de agua desde la boca probablemente presionando firmemente la lengua contra la mandíbula superior. Indudablemente la musculatura de la lengua juega un rol fundamental en la alimentación del delfín, ya que la lengua como destaca dicho autor empuja la comida hacia la orofarínge, orientando el pez para ser tragado siempre comenzando por la cabeza (probablemente para que las aletas y cola no se atoren). Finalmente el hecho de que los labios del delfín no sean móviles aunado a la presencia de papilas marginales en delfines jóvenes hace factible que la lengua sea vital en el proceso de succión.

La anatómica cardiaca macroscópica del corazón de T. truncatus coincide con la descripción hecha sobre el corazón del delfín del Plata (Pontoporia blainvillei) por Pérez \& Lima (2006) así como con la de los atlas de anatomía humana consultados para el presente estudio. Sin embargo destaca el caso del corazón \#2 en el que de la misma manera reseñada por Powell et al. (2009) se observa una transposición de la arteria pulmonar y la aorta, hipertrofia del ventrículo izquierdo, así como una pequeña conexión entre ambos atrios en forma de orificio (defecto ventricular septal) no perceptible en las fotografías exhibidas dentro de los resultados. Y como se menciona en dicho estudio existen pocas condiciones descritas en humanos y animales domésticos que expliquen anomalías cardiacas y vasculares múltiples; siendo una de estas condiciones la llamada Tetralogía de Fallot caracterizada por una aorta predominante, obstrucción del flujo del ventrículo derecho, defecto ventricular septal e hipertrofia del ventrículo derecho. Colectivamente estos defectos causan una reducción del flujo sanguíneo hacia los pulmones permitiendo a la vez la mezcla de sangre proveniente de ambos flancos del corazón, dando lugar a la afluencia de sangre pobre en oxígeno desde el corazón hacia el resto del cuerpo.

Alusivo al pulmón como ya mencionaba Berta et al. (2006) existen diferencias importantes entre los pulmones de mamíferos terrestres y marinos, así pues que la anatomía externa del pulmón de T. truncatus se equipara a la que Concha (2011) realizó sobre los pulmones de Phocoena spinipinnis, y del mismo modo que dicho autor indica para esta marsopa, el pulmón de T. truncatus tampoco presenta fisuras interlobulares 
en su superficie costal, dato que ya indicaban Berta et al. en algunos Pinípedos, sugiriendo una tendencia a reducir la lobulación. Lógicamente esta característica impidió identificar y señalar lóbulos en la descripción del presente estudio. Por otra parte hay que estimar lo sugerido por Piscitelli et al. (2013) acerca de que aparentemente el gran tamaño de los pulmones de delfínidos y focoenidos es una condición derivada que parece permitirles funcionar como un almacén de oxígeno para el intercambio gaseoso respiratorio de estos activos buceadores de aguas relativamente someras.

Lo reseñado acerca de la anatomía hepática de $T$. truncatus es similar a lo expuesto en los atlas de anatomía humana consultados.

La estructura anatómica del bazo de T. truncatus concuerda con la que Berta et al. realizaron con respecto a los Pinípedos y Cetáceos; así como con la reportada en los atlas de anatomía humana consultados para este estudio. Subrayando lo manifestado por Colangelo et al. relativo a la función del bazo en Cetáceos, que el autor señala como controvertida ya que el pequeño tamaño de este órgano, junto con su típica forma globular y superficie lisa, pueden argumentan en contra de su función de almacenamiento de glóbulos rojos oxigenados.

Los estómagos de los Cetáceos mantienen un sistema uniforme de morfología estomacal con las excepciones anotadas por Mead (1993) de las familias Ziphiidae (zifios) y Platanistidae (delfines de río). Por lo cual sobre el estómago del $T$. truncatus, el mismo autor señala que difiere únicamente en tamaño respecto al de una ballena azul (Balaenoptera musculus). Así pues en el caso del delfín nariz de botella, como en todos los cetáceos, se habla de un estómago plurilocular que consiste en: ante-estómago, estómago principal, cámaras de conexión y dos compartimentos pilóricos.

El delfín nariz de botella, como todos los mamíferos marinos, presenta un riñón reniculado tal como señalan Gaskin (1986), Lauria de Cidre \& Klein (1994) y Berta et al., lo que según estos últimos permite la producción de grandes volúmenes de orina concentrada. Para explicar esta característica debe considerarse lo indicado por Lauria de Cidre \& Klein al aseverar que los mamíferos marinos desarrollaron esta reniculación como un recurso evolutivo presionados por el medio en cuanto a la economía del agua, permitiéndoles albergar un mayor número de nefronas en un volumen renal menor, además de facilitar el funcionamiento independiente de cada renículo.

La anatomía externa del órgano reproductor femenino de T. truncatus corresponde con lo anotado por Meek
(1918) sobre la marsopa (Phocoena communis), por Berta et al. sobre los Pinípedos, así como con las descripciones generales sobre el útero no preñado en Cetáceos realizadas por Norris (1977) y Plön \& Bernard (2007). De lo anterior se observa que los órganos genitales son simples y típicos siguiendo el patrón general del útero bicorne en mamíferos. Sin embargo es de interés lo señalado por Meek acerca de la angosta entrada a la vagina que puede ser una adaptación para ocluir la entrada de agua hacia la cavidad de la vaginal.

Así pues, todos los resultados anatómicos estuvieron en conformidad con los de los autores presentados dentro de los antecedentes de este estudio, lo cual afianza el conocimiento de que los órganos internos de T. truncatus aquí descritos tipifican a los de los mamíferos en aspectos generales.

Cabe destacar lo mencionado por Daviau et al., sobre que el proceso de plastinación está actualmente en uso para la creación de modelos para escuelas médicas y veterinarias, aplicándose en una amplia variedad de especies, con la única limitación del tamaño de la cámara de impregnación; aunque la mayor desventaja radica en la necesidad de obtener licencia de von Hagens para utilizar la técnica patentada y obtener los materiales; motivo por el cual son necesarias más investigaciones adaptando la técnica original para crear muestras permanentes, de buena calidad, y de alto carácter ilustrativo con un costo implícito menor. Siendo el caso del presente trabajo con objeto de ayudar a la instrucción de la anatomía general de algunos órganos de T.truncatus.

SÁNCHEZ, F. G.; CONTRERAS, V. M. D. \& MORENO, C. R. Plastination and anatomy description of organs of the bottlenose dolphin (Tursiops truncatus). Int. J. Morphol., 34(2):644-652, 2016.

SUMMARY: Four different internal organs of bottlenose dolphin (Tursiops truncatus) were plastinated and described, obtaining didactic models, which let their components to be modified in an unaltered context; the resulting specimens are opaque and firm, but not unbreakable, with an appearance similar to that of the living state. The anatomical descriptions were made in concordance with those of the authors presented within the antecedents of this study; reinforcing the knowledge that the internal organs of T. truncatus described here typify those of mammals in general aspects, since the great uniformity between their structural elements is revealed. Moreover, there are very few non-pathological anatomic studies about dolphins, even though various mophometric and behavioral studies have been carried out, it still can be considered that several biological aspects of bottlenose dolphins remain to be described.

KEY WORDS: Plastination; Delphinidae; Bottlenose; Bodies; Description. 


\section{REFERENCIAS BIBLIOGRÁFICAS}

Ali, A. M. \& Al-Thnaian, T. A. Preservation of ruminant and equine anatomical specimens by silicone plastination. Sci. J. King Faisal Univ., 8(1):111-9, 2007.

Berta, A.; Sumich, J. L. \& Kovacs, K. M. Marine Mammals: Evolutionary Biology. $2^{\mathrm{a}}$ ed. San Diego, Academic Press, 2006.

Blaylock, R. A.; Hain, J. W.; Hansen, L. J.; Palka, D. L. \& Waring, G. T. U. S. Atlantic and Gulf of Mexico Marine Mammal Stock Assessments. NOAA Tech. Memo. NMFS-SEFSC-363, 1995.

Boya, J. Atlas de Histología y Organografía microscópica, $2^{\mathrm{a}}$ ed. Madrid, Panamericana, 2004.

Bravo, H. Plastination an additional tool to teach anatomy. Int. J. Morphol., 24(3):475-80, 2006

Colangelo, P.; Marruchella, G.; Cozzi, B.; Mazzariol, S.; García Hartmann, M.; Stanzani, G.; Fichtel, L.; Olivieri, V.; Albanese, V. \& Di Guardo, G. Accesory spleens in cetaceans: A morpho-pathological study in three bottlenose dolphins (Tursiops truncatus) and one Risso's dolphin (Grampus griseus). Poster en Congreso. Cattolica, Conferencia Internacional de Cetáceos, Tortugas Marinas y Tiburones del Mar Adriático, 2006.

Concha, I. Anatomical description of the lungs of Burmeister's porpoise (Phocoena spinipinnis). Santiago de Chile, Anato.cl, 2011. Disponible en: http://www.anato.cl/0007proyectos/porpoise/IConcha\%20AAVA \%20UST2011-porpoise.pdf

Castelló-Orvay, F. Morfología, Anatomía y Biología de los Cetáceos. Inmers. Cienc., (10-11):133-43, 1976.

Daviau, J. S.; Parker, J. C.; Parmelee, R. H.; Jahn, S. E. \& Frank, D. A. The use of plastinated specimens as teaching aids of orolaryngeal anatomy in selected laboratory animals. Contemp. Top. Lab. Anim. Sci., 36(6):50-2, 1997.

Donaldson, B. J. The tongue of the bottlenosed dolphin (Tursiops truncatus). In: Harrison, R. J. (Ed.). Functional Anatomy of Marine Mammals. Vol. 3. New York, Academic Press, 1977.

Fuentes Santoyo, R. Anatomía, Fisiología y Ciencias de la Salud. $4^{\mathrm{a}}$ ed. Ciudad de México, Editorial Trillas, 2006.

Gaskin, D. E. Distribution of Delphinidae (Cetacea) in relation to sea surface temperatures off eastern and southern New Zealand. N. Z. J. Mar. Freshwater Res., 2:527-34, 1968.

Kastelein, R. A. \& Dubbeldam, J. L. Marginal papillae on the tongue of the Harbour porpoise (Phocoena phocoena), Bottlenose dolphin (Tursiops truncatus) and Commerson's dolphin (Cephalorhynchus commersonii). Aquat. Mamm., 15(4):158-70, 1990.

Kularbkaewi, C.; Cook, P.; Yutanawiboonchai, W.; Von Hagens, G. Plastinated Pathology Specimens at Room Temperature in Thailand. J. Int. Soc. Plastination; 11(1):17-20, 1996.

Lauria de Cidre, L. \& Klein, M. Observaciones morfofuncionales en el riñón del lobo marino de un pelo Otaria flavescens. Misc. Zool., 17:213-23, 1993-1994.

Mead, J. G. The systematic importance of stomach anatomy in beaked whales. Int. Mar. Biol. Res. Instit. Rep., 4:75-86, 1993.
Mead, J. G. Stomach anatomy and use in defining systemic relationships of the Cetacean family Ziphiidae (beaked whales). Anat. Rec. (Hoboken), 290(6):581-95, 2007.

Meek, A. The Reproductive Organs of Cetacea. J. Anat., 52(Pt. 2):186-210, 1918

Miklosová, M. \& Miklos, V. Plastination with silicone method S 10--monitoring and analysis causes of failure. Biomed. Pap. Med. Fac. Univ. Palacky Olomouc Czech Repub., 148(2):237-8, 2004.

Norris, K. S. Whales, dolphins and porpoises. Berkeley, University of California Press, 1977.

Pérez, W. \& Lima, M. Cardiac Anatomy of the Pontoporia blainville. Int. J. Morphol., 24(3):351-6, 2006.

Piscitelli, M. A.; Raverty, S. A.; Lillie, M. A. \& Shadwick, R. E. A review of cetacean lung morphology and mechanics. J. Morphol., 274(12):1425-40, 2013.

Plön, S. \& Bernard, R. T. F. Anatomy with Particular Reference to the Female. In: Miller, D. L. (Ed.). Reproductive Biology and Phylogeny of the Cetacea: Whales, Dolphins and Porpoises. Enfield, Science Publishers, 2007. p.428.

Powell, J. W.; Archibald, R. T.; Cross, C. A.; Rotstein, D. S.; Soop, V. M. \& McFee, W. E. Multiple congenital cardiac abnormalities in an Atlantic bottlenose dolphin (Tursiops truncatus). J. Wildl. Dis., 45(3):839-42, 2009.

Reyes-Aguilar, M. E. Anatomía humana y plastinación. Bol. Mex. His. Fil. Med., 10(1):34-9, 2007

Ripani, M.; Bassi, A.; Perracchio, L.; Panebianco, V.; Perez, M.; Boccia, M. L.; Marinozzi G. Monitoring and enhancement of fixation, dehydration, forced impregnation and cure in the standard S-10 technique. J. Int. Soc. Plastination, 8:3-5, 1994.

Silva Pérez, E. Análisis de la Técnica de Plastinación Aplicada a Corazones de Cerdo. Tesis de Licenciatura (Biología). México D. F., Facultad de Estudios Superiores Iztacala, Universidad Nacional Autónoma de México, 2007. pp.42.

Sokolov, V. E. \& Volkova, O. V. Structure of the dolphin's tongue. In: Chapskii, K. K. \& Sokolov, V. E. (Eds.). Morphology and Ecology of Marine Mammals: Seals, Dolphins, Porpoises. New York, Wiley, 1973.

Vaughan, T. A. Mamíferos. 3a ed. Ciudad de México, Mc Graw-Hill, 1988.

von Hagens, G.; Tiedemann, K. \& Kriz, W. The current potential of plastination. Anat. Embryol. (Berl.), 175(4):411-21, 1987.

von Hagens, G. \& Whalley, A. Body Worlds: The Original Exhibition of Real Human Bodies. Catálogo de la Exhibición. 14a Reimpresión. Heidelberg, Verlagsgesellschaft mbH, 2009.

Dirección para Correspondencia:

Mtra. Gabriela Sánchez Fabila

FES Iztacala en el laboratorio de Anatomía de vertebrados y

Educación Científica LAVEC

Universidad Nacional Autónoma de México

Avenida de los Barrios Número 1

Colonia Los Reyes Iztacala

Tlalnepantla, Estado de México, C.P. 54090

MÉXICO

Email: gsfabila@gmail.com

Recibido : 05-11-2015

Aceptado: 13-04-2016 